\title{
Article \\ Consumption of Non-Nutritive Sweetener, Acesulfame Potassium Exacerbates Atherosclerosis through Dysregulation of Lipid Metabolism in ApoE ${ }^{-1-}$ Mice
}

\author{
Cheng-Hsin Lin ${ }^{1}$, Hung-Yuan Li ${ }^{2}\left(\mathbb{D}\right.$, Shu-Huei Wang ${ }^{3} \mathbb{D}$, Yue-Hwa Chen ${ }^{4,5}$, Yang-Ching Chen ${ }^{6,7}(\mathbb{D})$ \\ and Hung-Tsung $\mathrm{Wu}^{8, *(\mathrm{D})}$
}

Citation: Lin, C.-H.; Li, H.-Y.; Wang, S.-H.; Chen, Y.-H.; Chen, Y.-C.; Wu, H.-T. Consumption of Non-Nutritive Sweetener, Acesulfame Potassium Exacerbates Atherosclerosis through Dysregulation of Lipid Metabolism in ApoE $^{-/-}$Mice. Nutrients 2021, 13, 3984. https://doi.org/10.3390/ nu13113984

Academic Editors: Paolo Magni and Lindsay Brown

Received: 25 August 2021

Accepted: 4 November 2021

Published: 9 November 2021

Publisher's Note: MDPI stays neutral with regard to jurisdictional claims in published maps and institutional affiliations.

Copyright: (c) 2021 by the authors. Licensee MDPI, Basel, Switzerland. This article is an open access article distributed under the terms and conditions of the Creative Commons Attribution (CC BY) license (https:// creativecommons.org/licenses/by/ $4.0 /)$.
1 Department of Surgery, Shuang Ho Hospital, College of Medicine, Taipei Medical University, Taipei 110, Taiwan; chlin99025@tmu.edu.tw

2 Department of Internal Medicine, National Taiwan University Hospital, Taipei 100, Taiwan; larsli@ntuh.gov.tw

3 Department of Anatomy and Cell Biology, College of Medicine, National Taiwan University, Taipei 100, Taiwan; shwang@ntu.edu.tw

4 School of Nutrition and Health Sciences, Taipei Medical University, Taipei 110, Taiwan; yuehwa@tmu.edu.tw

5 School of Food Safety, Taipei Medical University, Taipei 110, Taiwan

6 Department of Family Medicine, Taipei Medical University Hospital, Taipei 110, Taiwan; melisa26@tmu.edu.tw

7 Department of Family Medicine, School of Medicine, College of Medicine, Taipei Medical University, Taipei 110, Taiwan

8 Department of Internal Medicine, School of Medicine, College of Medicine, National Cheng Kung University, Tainan 701, Taiwan

* Correspondence: z11008014@ncku.edu.tw; Tel.: +886-6-2353535 (ext. 5205); Fax: +886-6-2353660

Abstract: Obesity is associated with the risk of cardiovascular disease, and non-nutritive sweetener, such as acesulfame potassium (AceK) has been used to combat obesity. However, the effects of AceK on cardiovascular disease are still unclear. In this study, high cholesterol diet (HCD)-fed $\mathrm{ApoE}^{-/-}$mice had dysregulated plasma lipid profile, and developed atherosclerosis, determined by atherosclerotic plaque in the aorta. Supplement of AceK in HCD worsened the dyslipidemia and increased atherosclerotic plaque, as compared with $\mathrm{HCD}$-fed $\mathrm{ApoE}^{-/-}$mice. Since treatment of AceK in RAW264.7 macrophages showed no significant effects on inflammatory cytokine expressions, we then investigated the impacts of AceK on lipid metabolism. We found that AceK consumption enhanced hepatic lipogenesis and decreased $\beta$-oxidation in $\mathrm{ApoE}^{-/-}$mice. In addition, AceK directly increased lipogenesis and decreased $\beta$-oxidation in HepG2 cells. Taken together, a concurrent consumption of AceK exacerbated HCD-induced dyslipidemia and atherosclerotic lesion in ApoE ${ }^{-/-}$ mice, and AceK might increase the risk of atherosclerosis under HCD.

Keywords: acesulfame potassium; apolipoprotein E; atherosclerosis; dysregulation; lipid metabolism

\section{Introduction}

Obesity is a multifactorial disease that is linked with high prevalence rates of noncommunicable diseases [1-3]. The growing trend of obesity makes it as a serious public health threat that exhibits no boundaries [4]. The global prevalence of obesity has almost tripled since 1975 and obesity has a serious impact on human health [4]. Ample evidence has disclosed that sugar consumption is fueling the epidemic of obesity, elevating the risk of obesity-related morbidities [5]. Additionally, obesity contributes directly to the incidence of cardiovascular risk factors, including dyslipidemia, type 2 diabetes and hypertension [6]. Yet, cardiovascular disease is one of the leading causes of death globally [7]. Therefore, the public health issue of obesity should be taken seriously.

Recently, non-nutritive sweetener (NNS) has been widely used as a substitute for table sugar [8]. NNS is non-nutritive and contains no calories, attributes that could make it useful 
against obesity pandemic [9]. NNS is hundreds or thousands of times sweeter than table sugar and can provide a lingual sweet taste sensation without an excessive calorie load. These benefits have accelerated the use of NNS over the past decades [10,11]. In parallel to the dramatic increase in the consumption of NNS sweetened food, concerns have been raised about their potential adverse health effects. Several studies have proposed that NNS consumption may contribute to a profound impact on glucose homeostasis and insulin resistance, promoting excessive energy consumption from NNS-sweetened food products that even produce diet-induced obesity [11,12]. Other health hazards, such as brain tumor, cancer and dysbiosis of gut microbiota have also been proposed [12]. Thus, the health concerns are greater than the prospective beneficial effects of chronic NNS consumption.

Acesulfame potassium (AceK) is a chemically produced sweetener which belongs to one of five Food and Drug Administration-approved NNS that could be used as an additive in foods and beverages [13]. AceK is a heat-stable compound and highly soluble in water. It is 200 times sweeter than table sugar which is commonly used in baked goods, frozen desserts, candy, etc. $[8,12]$. Recently, some findings have suggested that chronic consumption of AceK plays a crucial role in intestinal glucose uptake and adipogenesis in murine models [11,14]. Cognitive impairment and genotoxicity effects induced by AceK consumption further challenge the safety use of AceK as a food additive [15-17]. Long-term consumption of AceK may induce hyperlipidemia in wild type mice [1]. However, the mechanism underlying the effects of AceK on atherosclerosis remains unknown. Thus, in the present study, we aimed to investigate the effects of AceK on the development of atherosclerosis in apolipoprotein $\mathrm{E}$ deficient $\left(\mathrm{ApoE}^{-/-}\right)$mice.

\section{Material and Methods}

\subsection{Experimental Animals}

Eight-week-old male ApoE ${ }^{-/-}$mice were purchased from National Laboratory Animal Center (Taipei, Taiwan) and housed in Laboratory Animal Center, Taipei Medical University, Taiwan under barrier-maintained conditions (temperature: $22 \pm 2{ }^{\circ} \mathrm{C}$, humidity: $55 \%, 12: 12 \mathrm{~h}$ light:dark cycle). The animals were randomly assigned to four groups ( $n=9$ per group): Group I (Chow), $\mathrm{ApoE}^{-/-}$mice fed with standard chow diet $(2.89 \mathrm{kcal} / \mathrm{g})$ (Laboratory Rodent Diet 5001, LabDiet, St. Louis, MO, USA); Group II (AceK), ApoE $\mathrm{Ap}^{-/-}$ mice fed with chow diet and AceK; Group III (high cholesterol diet, HCD), ApoE ${ }^{-/-}$ mice fed with HCD; Group IV (HCD-AceK), $\mathrm{ApoE}^{-/-}$mice fed with HCD and AceK. The HCD contained $21 \%$ fat and $0.15 \%$ cholesterol $(4.67 \mathrm{kcal} / \mathrm{g}$ ) (D12079B, Research diet, New Brunswick, NJ, USA) and was given for 8 weeks to accelerate the development of atherosclerosis in $\mathrm{ApoE}^{-/-}$mice. AceK (Sigma-Aldrich, St. Louis, MO, USA) of $15 \mathrm{mg} / \mathrm{kg}$ body weight per day, which is equal to the acceptable daily intake (ADI), was dissolved in sterile saline and administrated by oral gavage once daily [18]. The food intake was measured weekly and converted into caloric intake.

\subsection{Morphological Analysis}

The heart and aortic tissue were harvested and fixed with $10 \%$ formalin according to a previous study [19]. The tissue was embedded in optimal cutting temperature compound and cut into $3 \mu \mathrm{m}$ sections for histological measurement of atherosclerotic lesions in aortic sinus. Oil red O staining (Sigma-Aldrich, St. Louis, MO, USA) was used to visualize the atherosclerotic lesion area. Lesion area was quantified using imageJ software on 12 July 2020 (https://imagej.nih.gov/ij/).

\subsection{Biochemical Analysis}

The mice were fasted for $12 \mathrm{~h}$ and then blood samples were collected for the determination of lipid profile using commercialized assay kits. Serum triglyceride concentrations were measured using triglyceride colorimetric assay kit (Cayman, Ann Arbor, MI, USA) and total cholesterol, low-density lipoprotein cholesterol (LDL-cholesterol) and high-density 
lipoprotein cholesterol (HDL-cholesterol) were measured using EnzyChrom ${ }^{\mathrm{TM}}$ AF HDL and LDL/VLDL assay kits (Bioassay System, Hayward, CA, USA).

\subsection{Cell Culture}

Mus musculus macrophage cell line (RAW264.7) and Homo Sapiens hepatocellular carcinoma cell line (HepG2) were purchased from the Bioresource Collection and Research Center (Hsinchu, Taiwan). RAW264.7 murine macrophage cells were grown in $25 \mathrm{~cm}^{2}$ flasks in RPMI-1640 medium containing 10\% fetal bovine serum with penicillin $(100 \mathrm{U} / \mathrm{mL})$ and streptomycin $(100 \mu \mathrm{g} / \mathrm{mL})$. HepG2 cells were maintained in Dulbecco's Modified Eagle Medium (Gibco, Amarillo, TX, USA) with 10\% fetal bovine serum and 100 units $/ \mathrm{mL}$ penicillin, and $100 \mu \mathrm{g} / \mathrm{mL}$ streptomycin.

\subsection{Real-Time Polymerase Chain Reactions}

The cells were subjected to ribonucleic acid (RNA) isolation using GENEzol ${ }^{\mathrm{TM}}$ TriRNA Pure Kit (Geneaid Biotech, New Taipei City, Taiwan). Two $\mu$ g of RNA were used to react with Moloney murine leukemia virus reverse transcriptase (ProTech, Taipei, Taiwan) to generate complementary deoxyribonucleic acid. Polymerase chain reaction (PCR) amplification was performed using the target primers and Luna Universal qPCR Master Mix (New England Biolabs, Ipswich, MA, USA) for $10 \mathrm{~min}$ at $95^{\circ} \mathrm{C}$, followed by 40 cycles set for $10 \mathrm{~s}$ at $95^{\circ} \mathrm{C}$, annealing for $10 \mathrm{~s}$ at $65^{\circ} \mathrm{C}$, and extending for $2 \mathrm{~s}$ at $72{ }^{\circ} \mathrm{C}$. Glyceraldehyde 3-phosphate dehydrogenase (GAPDH) was used as an internal control. The sequences of the target primers were shown as following. Mus musculus tumor necrosis factor- $\alpha$ (Tnf): forward: $5^{\prime}$ - GGT GCC TAT GTC TCA GCC TCT T $-3^{\prime}$, reverse: $5^{\prime}$ - GCC ATA GAA CTG ATG AGA GGG AG-3'; Mus musculus interleukin 6 (Il-6): forward: 5'-TAC CAC TTC ACA AGTCG GAG GC- $3^{\prime}$, reverse: $5^{\prime}$ - CTG CAA GTG CAT CAT CGT TGT TC- $3^{\prime}$; Mus musculus C-C Motif Chemokine Ligand 2 ( $C c l 2$ ): forward: 5'- GCT ACA AGA GGA TCA CCA GCA G-3', reverse 5'-GTC TGG ACC CAT TCC TTC TTG G-3'; Mus musculus Gapdh: 5'- CAT CAC TGC CAC CCA GAA GAC TG-3' ${ }^{\prime}$, reverse: $5^{\prime}$ - ATG CCA GTG AGC TTC CCG TTC AG-3'. Homo sapiens acetyl-coA carboxylase (ACC): forward: 5'-ACA TTC CCT GAG GCA GGT CA-3', reverse: 5'-GAT CCC ATG GTC AAC CAG GG-3'; Homo sapiens fatty acid synthase (FASN): forward: 5'-CGG TGT GTG CTG CTC TCC AA-3', reverse: 5'-CAG CAG GAA GTG GCG GAA AG-3'; Homo sapiens sterol regulatory element binding protein-1 (SREBP1): forward: $5^{\prime}$-GGG CCT TGC ATT TTC TGA CA-3', reverse: 5'-CAC GAA GAA ACG GTG GCC CA-3'; Homo sapiens peroxisomal acyl-coenzyme A oxidase (ACOX): forward: $5^{\prime}$-GGC GCA TAC ATG AAG GAG ACC T-3', reverse: 5'-AGG TGA AAG CCT TCA GTC CAG C-3'; Homo sapiens carnitine palmitoyltransferase 2 (CPT2): forward: $5^{\prime}-$ CCCTGCATACCAGCGGATAA-3' ${ }^{\prime}$, reverse: $5^{\prime}$-CATACGCAATGCCAAAGCCA-3'; Homo sapiens peroxisome proliferator-activated receptor- $\alpha(P P A R A)$ : forward: $5^{\prime}$-GCC TGT CTG TCG GGA TGT-3', reverse: 5'-GGC TTC GTG GAT TCT CTT G-3'; 3-hydroxy-3-methylglutaryl-coenzyme A reductase (HMGCR): forward: $5^{\prime}$-GAC GTG AAC CTA TGC TGG TCA G-3', reverse: 5'-GGT ATC TGT TTC AGC CAC TAA GG-3'; Homo sapiens GAPDH: 5'-TGG AAA TCC CAT CAC CAT CT-3', reverse: 5'-GTC TTC TGG GTG GCA GTG AT-3'

\subsection{Western Blot Analysis}

The samples were lysed using radioimmunoprecipitation assay buffer containing protease inhibitors (Sigma-Aldrich, St. Louis, MO, USA). Protein concentrations were determined using bicinchoninic acid protein assay (G-Biosciences, Maryland Heights, MO, USA). Thirty $\mu \mathrm{g}$ of protein lysates were separated using sodium dodecyl sulfatepolyacrylamide gel electrophoresis and transfer onto polyvinylidene fluoride membrane (Millipore, Burlington, MA, USA). The membranes were blocked at room temperature for one hour using TBS-T (10 mM Tris, $150 \mathrm{mM} \mathrm{NaCl}$, and $0.05 \%$ Tween 20, (pH 7.6)) containing 10\% skimmed milk, then probed with 1:1000 primary antibodies, such as FAS (Abcam, Cambridge, UK), ACC (Cell signaling, Danvers, MA, USA), SREBP1 (Novus, Centennial, CO, USA), ACOX1, CPT2 (Affinity Biosciences, Pottstown, PA, USA), PPAR $\alpha$ (GeneTex, 
Alton Pkwy Irvine, CA, USA), HMGCR (Abcam, Cambridge, UK) and actin (Abnova, Taipei, Taiwan) at $4{ }^{\circ} \mathrm{C}$ overnight. Blots were then washed with TBS-T and incubated with a 1:5000 dilution of horseradish peroxidase-conjugated secondary antibodies (Abcam, Cambridge, UK) at room temperature for one hour. Protein bands were visualized using a chemiluminescence horseradish peroxidase substrate (Millipore, Burlington, MA, USA), and the relative signal intensity was quantified using ImageJ software.

\subsection{Statistical Analysis}

GraphPad Prism 6 was used for all statistical analyses. Data was analyzed using unpaired Student's $t$-test and presented as the mean \pm standard error of the mean (SEM), and significance levels were indicated at $p<0.05$.

\section{Results}

\subsection{AceK Exacerbated Atherosclerosis in High Cholesterol Diet Fed ApoE ${ }^{-/-}$Mice}

After an eight-week feeding of HCD with or without AceK, body weight showed a significantly increase in HCD group, as compared with Chow group, whereas there were no significant differences between HCD group, and HCD-AceK group (Figure 1A). In addition, we found a significant decrease of daily calorie intake in HCD-AceK group (Figure 1B). To determine the effects of AceK on the development of atherosclerosis, we then measured the atherosclerotic plaque formed in aortic sinus. It was known that $\mathrm{HCD}$ accelerated the development of atherosclerosis, as compared with chow diet in $\mathrm{ApoE}^{-/-}$ mice. In this study, we found mild atherosclerotic plaque in chow-fed $\mathrm{ApoE}^{-1-}$ mice at the age of sixteen-weeks-old. However, a notably atherosclerotic plaque was formed in the aortic sinus in $\mathrm{HCD}$-fed $\mathrm{ApoE}^{-/-}$mice. AceK intervention further exacerbated the development of atherosclerosis (Figure 1C,D). We therefore examined the aortic sinus lesion area in both groups of $\mathrm{HCD}$-fed $\mathrm{ApoE}^{-/-}$mice and $\mathrm{HCD}$-fed AceK supplemented $\mathrm{ApoE}^{-/-}$mice (Figure 1D). The aortic sinus lesion area was significantly increased in HCD-fed AceK supplemented $\mathrm{ApoE}^{-/-}$mice, as compared with HCD-fed mice, indicating AceK may accelerate the development of atherosclerosis.

A
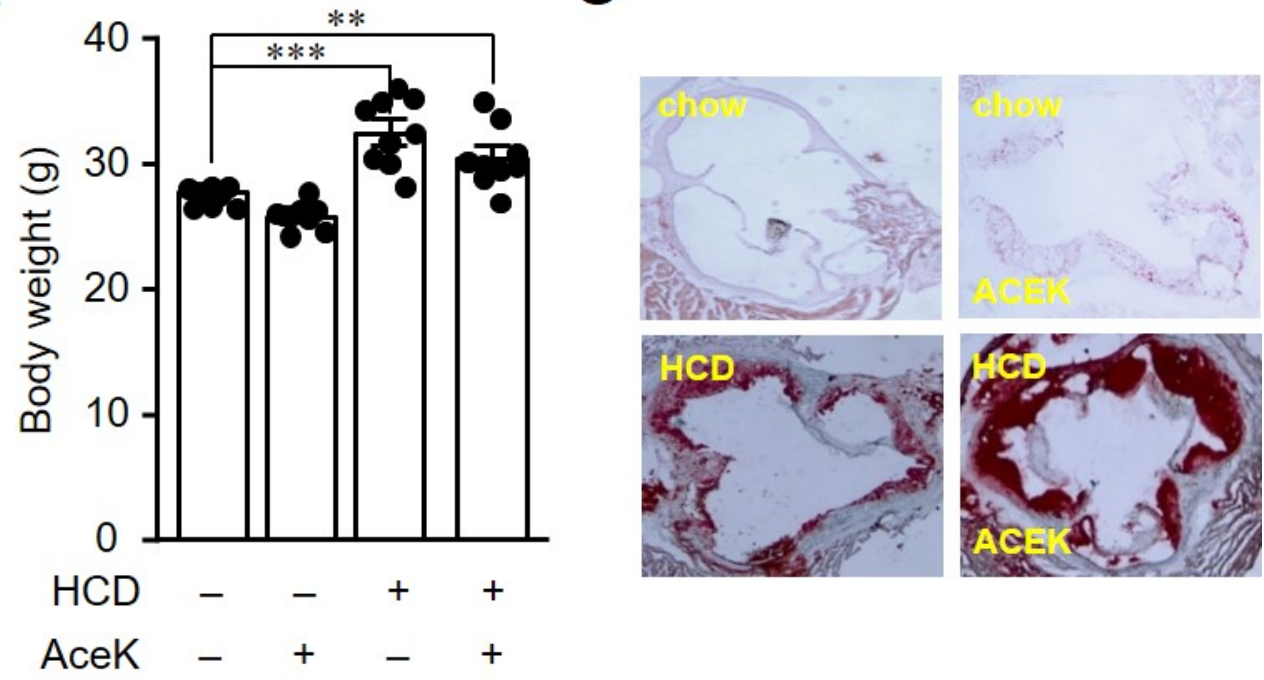

Figure 1. Cont. 
B

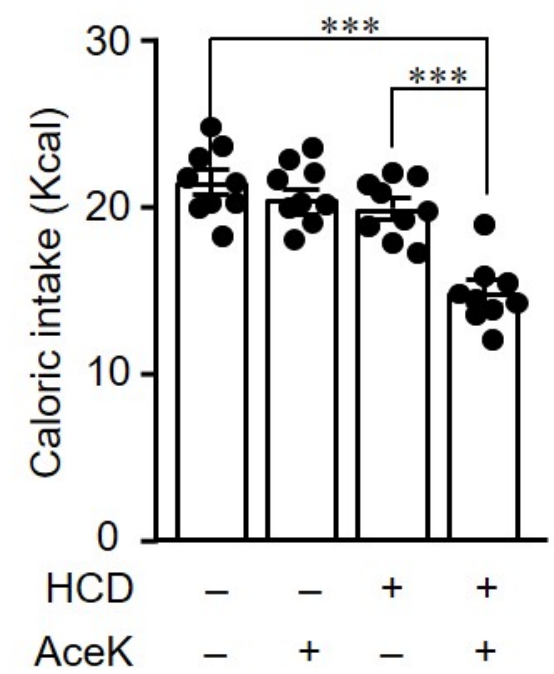

D

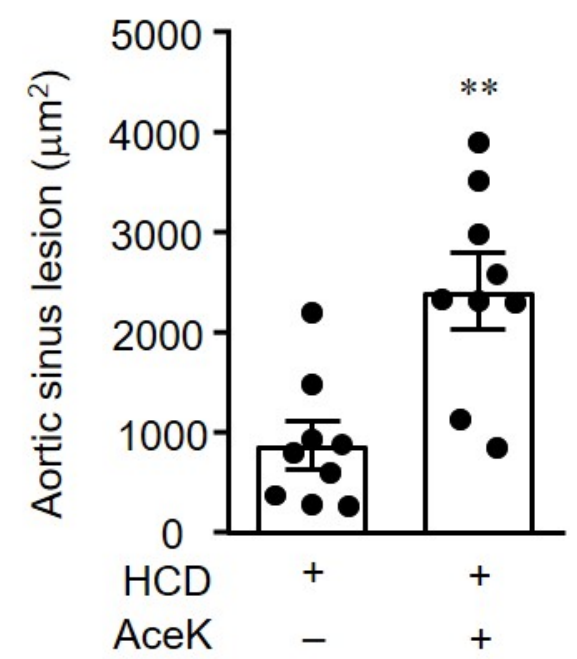

Figure 1. AceK exacerbated atherosclerosis in high cholesterol diet-fed $\mathrm{ApoE}^{-/-}$mice. Mice were fed with chow diet or high cholesterol diet (HCD) for 8 weeks with or without $15 \mathrm{mg} / \mathrm{kg}$ AceK administration once daily. The body weight (A), and calorie intake (B) were recorded. The aortic sinus sections were stained with Oil Red $\mathrm{O}$ to visualize the atherosclerotic formed (C), and the quantification of the aortic sinus lesion area by imageJ (D). ${ }^{* *} p<0.01,{ }^{* * *} p<0.001$.

3.2. AceK Showed No Significant Effects on Proinflammatory Cytokine Expressions in RAW264.7 Macrophages

The underlying pathogenesis of atherosclerosis encompassed an imbalanced lipid metabolism and a maladaptive immune response entailing a chronic inflammatory response in the arterial wall. The persistent inflammatory signals further lead to an endothelial dysfunction. We therefore investigated the inflammatory cytokine expressions in responses to AceK treatment in murine macrophages. As shown in Figure 2, treatment of AceK at various doses in RAW264.7 for $24 \mathrm{~h}$, the expressions of Tnfa (Figure 2A), Ccl2 (Figure 2B) and Il-6 (Figure 2C) showed no significant differences between AceK treated and untreated groups, implying inflammation might not be involved in AceK-accelerated atherosclerosis.

A

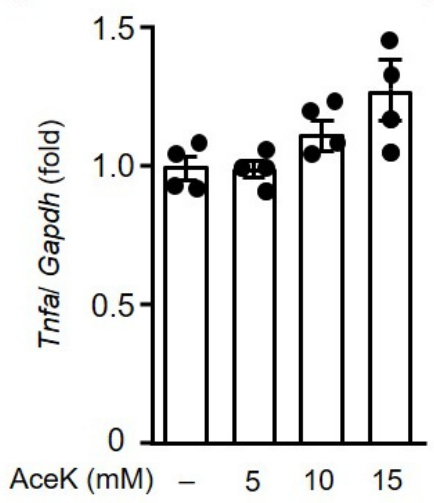

B

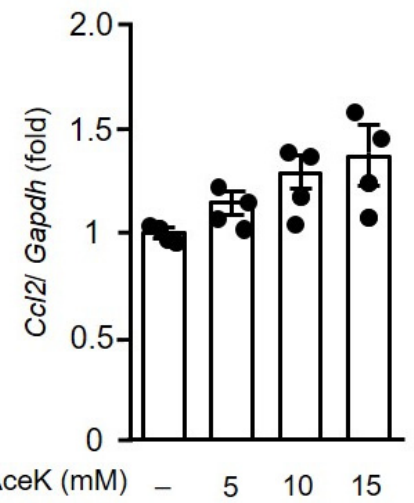

C

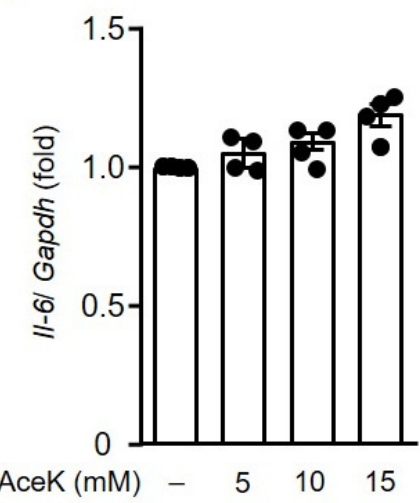

Figure 2. Effects of AceK on proinflammatory cytokine expressions in murine RAW264.7 macrophages. Cells were treated with indicated doses of AceK for $24 \mathrm{~h}$. The cells were harvested and RNA was isolated for the quantification of tumor necrosis factor alpha (Tnfa) (A), C-C motif chemokine ligand 2 $(\mathrm{Ccl})(\mathbf{B})$, and interleukin-6 (Il-6) (C) gene expressions by quantitative PCR $(n=4)$. 


\subsection{AceK Worsened Dyslipidemia in High Cholesterol Diet-Fed ApoE ${ }^{-/-}$Mice}

Accumulating studies disclosed that dyslipidemia can provoke endothelial dysfunction by aggregating immune cells on the arterial wall [20]. In order to investigate the effects of AceK on lipid homeostasis, we then analyzed the lipid profile in $\mathrm{ApoE}^{-/-}$ mice. Consistent with previous study, we found that AceK treatment slightly, but significantly, induced hyperlipidemia [1]. In addition, the plasma total cholesterol (Figure ??A), triglycerides (Figure ??B) and LDL-cholesterol (Figure ??C) were significantly increased in $\mathrm{ApoE}^{-/-}$mice, whereas HDL-cholesterol levels were decreased (Figure ??D), as compared with Chow group. AceK intervention further increased the total cholesterol, triglyceride and LDL-cholesterol concentrations than HCD group, whereas the levels of HDL-c were further decreased (Figure ??). Together with the morphological analysis, we suggested that AceK exacerbated the development of atherosclerosis through the dysregulation of lipid metabolism.

A

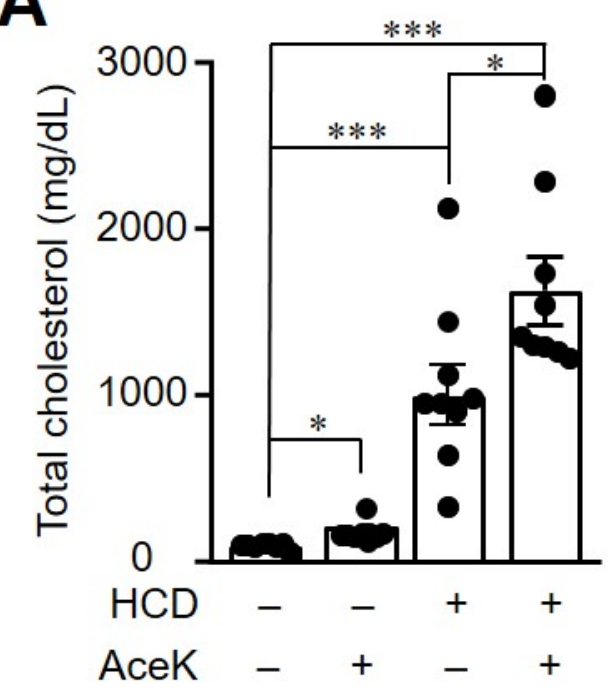

C

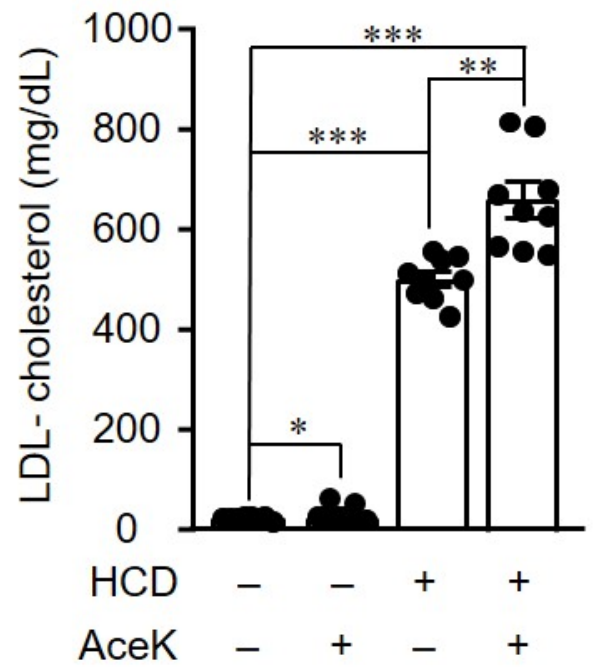

B

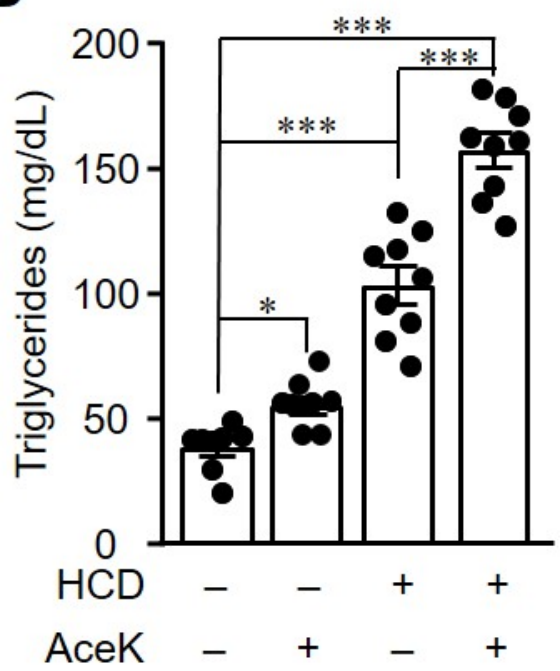

D

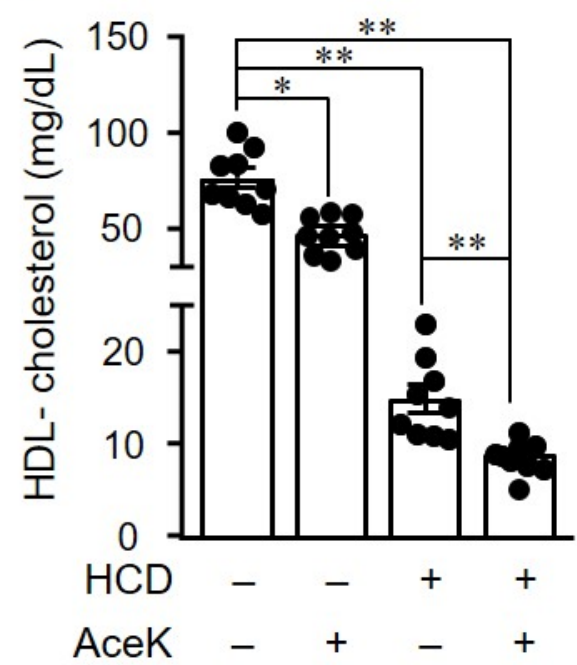

Figure 3. Administration of AceK worsened dyslipidemia in high cholesterol diet-fed $\mathrm{ApoE}^{-/-}$ mice. The mice were fasted for $12 \mathrm{~h}$ and then blood samples were collected for the determination of lipid profiles. Plasma total cholesterol (A), triglyceride (B), low-density lipoprotein cholesterol (LDLcholesterol) (C), and high-density lipoprotein cholesterol (HDL-cholesterol) (D) were determined using commercialized assay kits $\left(n=9\right.$ per group of mice). ${ }^{*} p<0.05,{ }^{* *} p<0.01,{ }^{* * *} p<0.001$. 


\subsection{AceK Impaired Lipid Homeostasis in ApoE ${ }^{-/-}$Mice}

To clarify the possible mechanisms related to lipid metabolic dysregulation induced by AceK, we investigated the key proteins in lipogenesis and lipolysis pathways. As shown in Figure 4, the lipogenesis-related proteins, such as ACC, FAS and SREBP1 protein expressions were significantly increased in HCD group as compared with Chow group. AceK intervention further significantly increased the hepatic expressions of ACC, FAS and SREBP1 than HCD group. In addition, we found that the expression of the rate-limiting enzyme for cholesterol synthesis, HMGCR, was significant decreased in HCD-fed ApoE ${ }^{-/-}$ mice, and AceK supplement further enhanced the effects of HCD on HMGCR expression
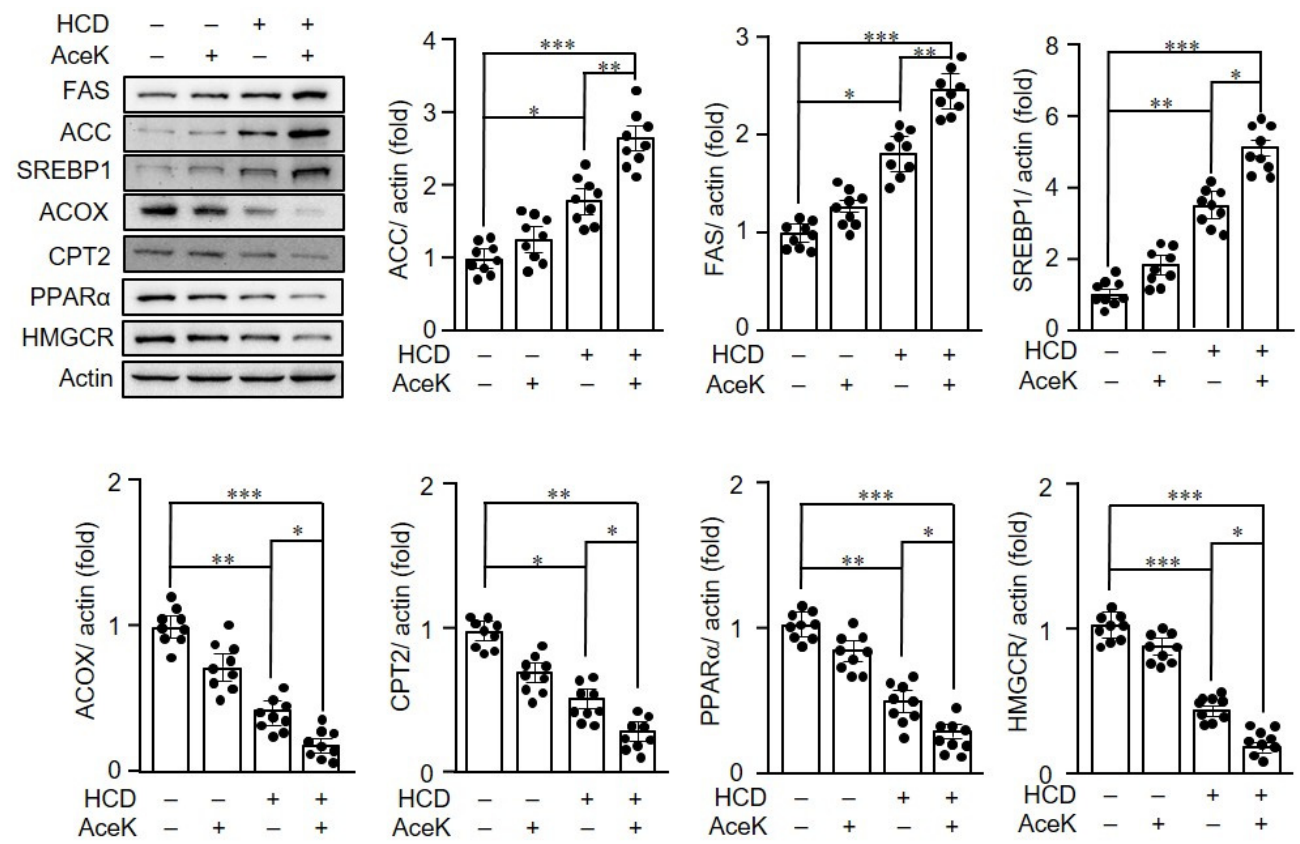

Figure 4. AceK dysregulated lipid homeostasis in $\mathrm{ApoE}^{-/-}$mice. The protein expressions in the liver of $\mathrm{ApoE}^{-/-}$mice of acetyl-coA carboxylase (ACC), fatty acid synthase (FAS), sterol regulatory element binding protein-1 (SREBP1), peroxisomal acyl-coenzyme A oxidase (ACOX), carnitine palmitoyltransferase-2 (CPT2), peroxisome proliferator-activated receptor $\alpha$ (PPAR $\alpha)$ and 3-hydroxy3-methyl-glutaryl-coenzyme A reductase (HMGCR) were determined by Western blots ( $n=9$ per group of mice). ${ }^{*} p<0.05,{ }^{* *} p<0.01,{ }^{* * *} p<0.001$.

Lipid homeostasis is a subtle balance between lipogenesis and lipolysis [21]. Thus, we further measured the hallmark proteins in lipolysis. HCD group showed significantly decreased ACOX, CPT-2, and PPAR $\alpha$ expressions than Chow group. The AceK intervention further augmented the effects of HCD on the hepatic protein expressions of ACOX, CPT2 and PPAR $\alpha$.

\subsection{AceK-Induced Dysregulation of Lipid Homeostasis in HepG2 Cells}

Following the investigation of the effects of AceK on lipid metabolism in animals, we then elucidated whether AceK directly induced the imbalance of lipid homeostasis. HepG2 cells were used and treated with different doses of AceK. AceK treated-HepG2 cells showed significant upregulation of the lipogenesis-related gene expressions, including ACC (Figure 5A), FASN (Figure 5B) and SREBP1 (Figure 5C). On the other hand, AceK treatment in HepG2 cells showed significant lower lipolysis-related gene expressions, including ACOX (Figure 5D), CPT2 (Figure 5E) and PPAR $\alpha$ (Figure 5F) than control group. To further confirm the effects of AceK on lipid metabolism, we analyzed lipogenic and lipolytic protein expressions in AceK-treated HepG2 cells. In accordance with the results of lipogenic and lipolytic gene expressions, we found that AceK treatment dose-dependently 
increased lipogenesis-related protein expressions, and decreased lipolysis-related protein expressions (Figure 6).

A

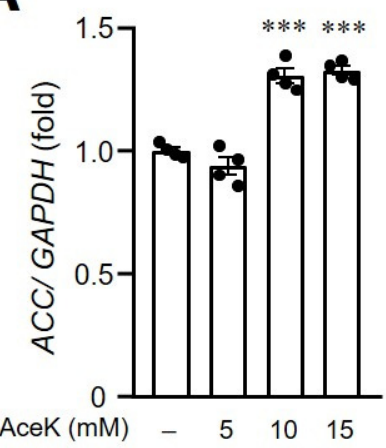

D

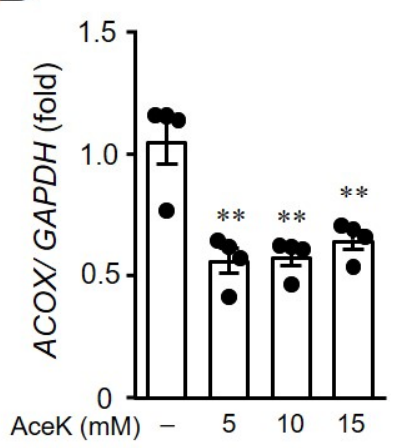

B

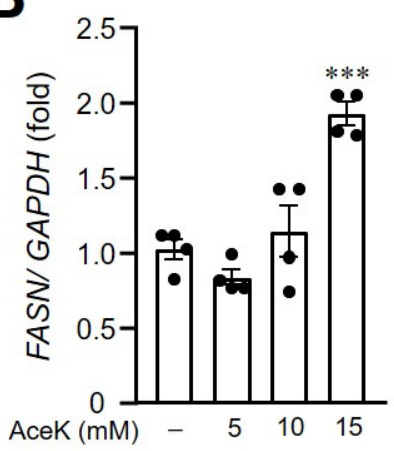

E

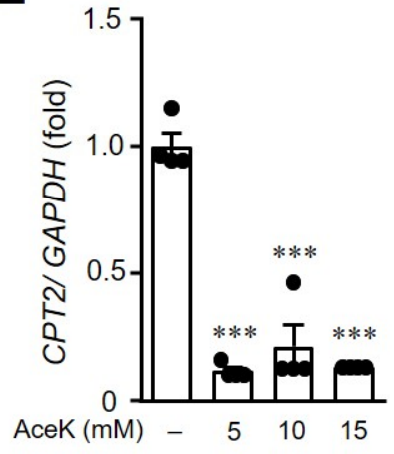

C

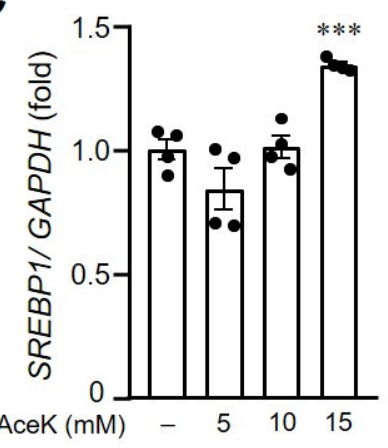

$\mathbf{F}$

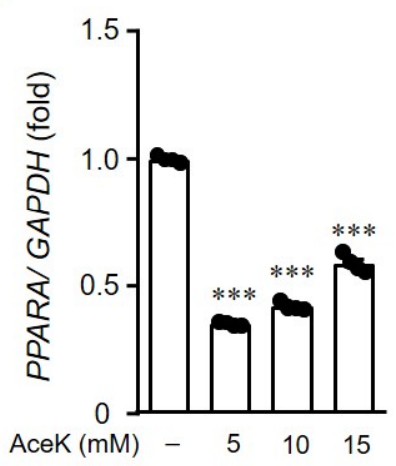

\section{G}

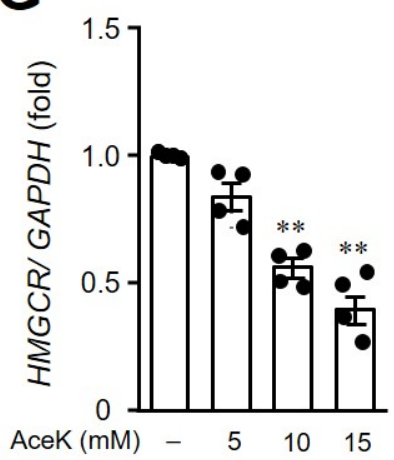

Figure 5. Effects of AceK on lipid metabolism-related gene expressions in HepG2 cells. HepG2 were treated with indicated doses of AceK for $24 \mathrm{~h}$. The cells were then harvested and RNA was isolated for the quantification of acetyl-coA carboxylase (ACC) (A), fatty acid synthase (FASN) (B), sterol regulatory element binding protein-1 (SREBP1) (C), peroxisomal acyl-coenzyme A oxidase $(A C O X)(\mathbf{D})$, carnitine palmitoyltransferase-2 (CPT2) $(\mathrm{E})$, peroxisome proliferator-activated receptor- $\alpha$ $(P P A R A)(\mathbf{F})$, and 3-hydroxy-3-methyl-glutaryl-coenzyme A reductase (HMGCR) (G) gene expressions by quantitative PCR $(n=4){ }^{* *} p<0.01,{ }^{* * *} p<0.001$. 

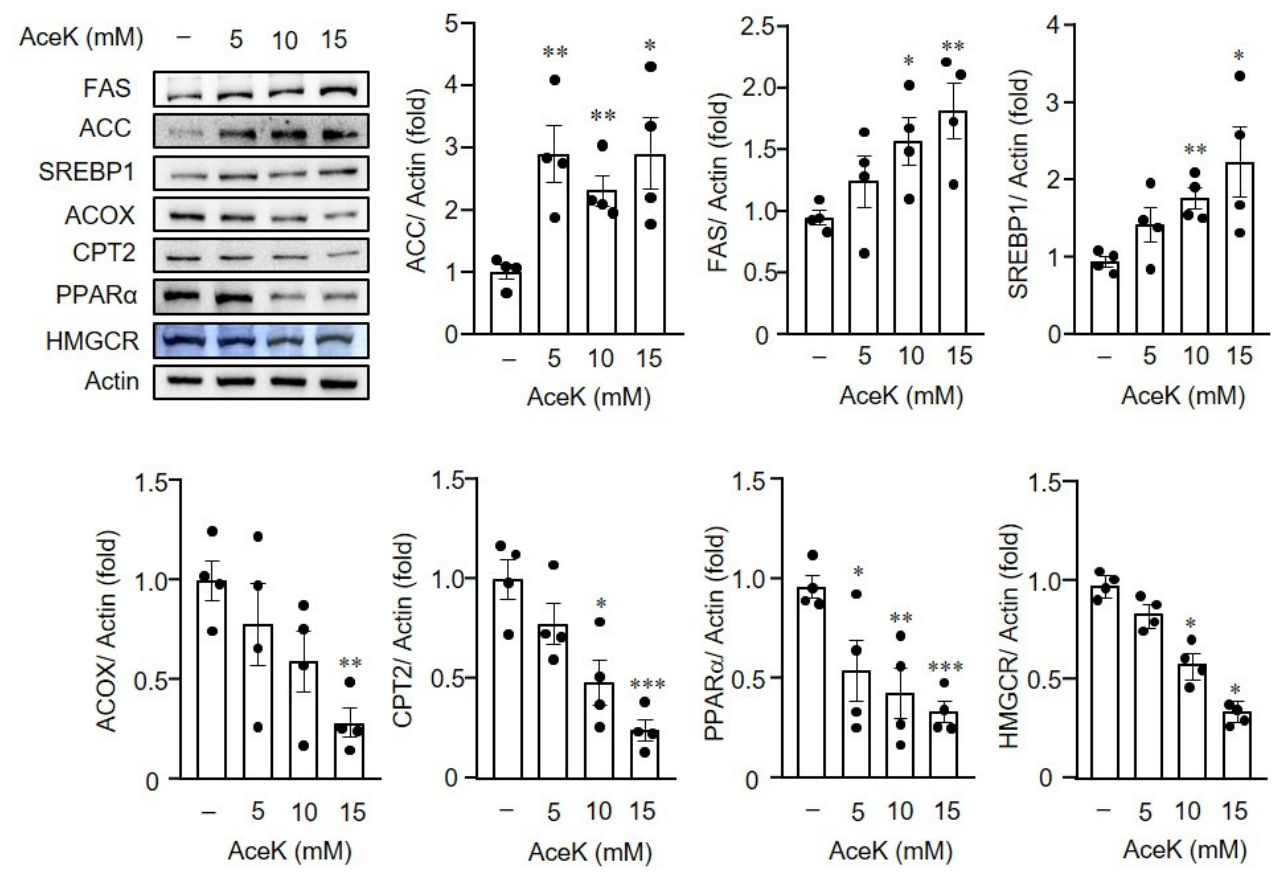

Figure 6. Effects of AceK on lipid metabolism-related protein expressions in HepG2 cells. HepG2 were treated with indicated doses of AceK for $24 \mathrm{~h}$. The protein lysates were prepared for the quantification of acetyl-coA carboxylase (ACC), fatty acid synthase (FAS), sterol regulatory element binding protein-1 (SREBP1), peroxisomal acyl-coenzyme A oxidase (ACOX), carnitine palmitoyltransferase-2 (CPT2), peroxisome proliferator-activated receptor $\alpha(\operatorname{PPAR} \alpha)$ and 3-hydroxy-3-methyl-glutarylcoenzyme A reductase (HMGCR) protein expressions by Western blots $(n=4)$. ${ }^{*} p<0.05,{ }^{* *} p<0.01$, *** $p<0.001$.

\section{Discussion}

To the best of our knowledge, the present study is the first report to investigate the effects of non-nutritive sweetener, AceK on the mechanism underlying the pathogenesis of atherosclerosis. In this study, we found that AceK consumption might exacerbate HCDinduced atherosclerosis. AceK significantly increased the blood lipid levels in $\mathrm{ApoE}^{-/-}$ mice, which led to a severe hyperlipidemia. Moreover, an upregulation of lipogenesisrelated genes alongside with a downregulation of $\beta$-oxidation-related genes resulted in an imbalance of lipid homeostasis. These effects of AceK on lipid metabolism might further augment the severity of atherosclerosis.

In accordance to the previous study, a notably atherosclerotic plaque was formed in the aortic sinus and aorta in $\mathrm{HCD}-\mathrm{fed} \mathrm{ApoE}^{-/-}$mice [22]. In the present study, we disclosed an additional effect of AceK on the progression of atherosclerosis. We found that there were no significant differences of body weight between HCD group, and HCD AceK group, consistent with a previous study indicating that chronic ingestion of AceK has limited influence on body weight and metabolic homeostasis in C57BL/6 mice [16]. However, the calorie intake of HCD-AceK group was significantly lower than that of HCD group in $\mathrm{ApoE}^{-/-}$mice without significant body weight change, implying AceK might have effects on lipid metabolism.

Atherosclerosis is a chronic inflammation and immune disease, characterized by a dysfunctional interplay between the impaired immunity and dyslipidemia [23]. Inflammationinduced endothelial dysfunction is the early key marker for atherosclerosis. We thereby treated RAW264.7 macrophages with AceK to integrate the effects of AceK on inflammation. In the present study, our results demonstrated that AceK had no significant effects on inflammatory responses in macrophages. In view of a significant higher atherosclerotic lesion area in aortic sinus after AceK consumption, we then further investigated the effects of AceK on lipid homeostasis. 
Sweet taste receptors are not only the receptors sensing sweetness, but play important roles in the regulation of lipid metabolism. It was shown that both T1R2 and T1R3 knockout mice have reduced adiposity and smaller adipocytes [24], implying activation of the sweet taste receptors might facilitate lipogenesis. AceK is one of the ligands that binds to sweet taste receptors [25], and extracellular signal-regulated kinase mitogen-activated protein kinase (ERK1/2)-pathway is one of main downstream signals for sweet taste receptors [26]. Previous studies found that the activation of ERK1/2 pathway decreased cardiac PPAR $\alpha$ gene expression and activity [27]. Although multiple mechanisms might be involved in artificial sweetener-stimulated adipogenesis and -suppressed lipolysis [28], we speculated that ACEK might regulate the levels of PPAR $\alpha$ through activation of the sweet taste receptors, and further studies are needed to investigate the detail mechanisms related to ACEK-induced hyperlipidemia.

Ample studies have demonstrated that abnormal lipid homeostasis may overtly elevate atherosclerotic risks [29]. In the present study, a dramatic increase in plasma total cholesterol, triglyceride and LDL-cholesterol concentrations were found in $\mathrm{ApoE}^{-/-}$mice fed with HCD, which conformed with the findings of previous studies [30]. Deletion of the ApoE gene caused an inability in clearance of circulatory lipid and even a boost in the sensitivity to a dietary cholesterol, making the mice suffer a severe hypercholesterolemia [30]. In addition, we found that AceK might regulate the lipid metabolism, including lipogenesis and lipolysis to exacerbate hyperlipidemia in HCD AceK group. On the other hand, a study indicated that NNS consumption may be attributable to endocrine metabolism [13]. Inhibition of cholesterol clearance by ApoE knockout showed a feedback effect to decrease HMGCR expressions in $\mathrm{ApoE}^{-/-}$mice [31,32]. In the present study, we found that AceK treatment decreased the expressions of HMGCR, consistent with a previous study indicating that AceK might alter the structure of HDL-cholesterol, and decrease the binding ability of the lipoprotein, thereby affecting the lipid clearance activity in the body [33]. Thus, not only the effects of Acek on lipogenesis and lipolysis might be related to the AceK-exacerbated atherosclerotic, but also the cholesterol clearance activity.

Although $\mathrm{ApoE}^{-/-}$mice are extensively used for atherosclerosis research, there are still several limitations [34,35], and the model does not compare well to human in terms of lipid profile. Thus, the effects of AceK on the development of atherosclerosis in human still need further studies to investigate.

\section{Conclusions}

Taken together, we found that AceK augmented HCD-induced dyslipidemia through an increment in lipogenesis and decrement in lipolysis, and these effects might further exacerbate $\mathrm{HCD}$-induced atherosclerosis in $\mathrm{ApoE}^{-/-}$mice. Thus, AceK posed additional effects on lipogenesis alongside with HCD. AceK might disturb lipid metabolism in the liver which further contributes to lipid dysregulation thereby enhancing the progression of atherosclerosis.

Author Contributions: C.-H.L. designed and performed the experiments, analyzed the data, and wrote the manuscript. C.-H.L. and H.-Y.L. wrote the manuscript and edited the text. S.-H.W., Y.-H.C. and Y.-C.C. provided technical assistance. H.-T.W. designed and supervised the study and wrote the manuscript. All authors have read and agreed to the published version of the manuscript.

Funding: This research was funded by the Ministry of Science and Technology, Taiwan (grant number: 110-2314-B-006-116-MY3 and 110-2314-B-006-115-). The APC was funded by the Ministry of Science and Technology, Taiwan.

Institutional Review Board Statement: All animal experiments were approved by the Institutional Animal Care and Use Committee of Taipei Medical University (LAC-2020-0350) and performed in accordance with National Institute of Health guide for the care and use of laboratory animals.

Informed Consent Statement: Not applicable.

Data Availability Statement: Data is contained within the article. 
Acknowledgments: We gratefully acknowledge $\mathrm{Ng}$ Xin Er for technical assistance related to the sections of aortic sinus.

Conflicts of Interest: The authors declare no conflict of interest.

$\begin{array}{ll}\text { Abbreviations } \\ \text { AceK } & \text { Acesulfame potassium } \\ \text { ACC } & \text { Acetyl-coA carboxylase } \\ \text { ApoE } & \text { Apolipoprotein E deficient } \\ \text { CPT2 } & \text { Carnitine palmitoyltransferase-2 } \\ \text { FAS } & \text { Fatty acid synthase } \\ \text { GAPDH } & \text { Glyceraldehyde 3-phosphate dehydrogenase } \\ \text { HCD } & \text { High cholesterol diet } \\ \text { HUVECs } & \text { Human umbilical vein endothelial cell line } \\ \text { HDL-c } & \text { High-density lipoprotein cholesterol } \\ \text { IL-6 } & \text { Interleukin 6 } \\ \text { LDL-c } & \text { Low-density lipoprotein cholesterol } \\ \text { CCL2 } & \text { C-C motif chemokine ligand 2 } \\ \text { NNS } & \text { Non-nutritive sweetener } \\ \text { ACOX } & \text { Peroxisomal acyl-coenzyme A oxidase } \\ \text { PPAR } \alpha & \text { Peroxisome proliferator-activated receptor- } \alpha \\ \text { SREBP1 } & \text { Sterol regulatory element binding protein-1 } \\ \text { TC } & \text { Total cholesterol } \\ \text { TG } & \text { Triglycerides } \\ \text { TNF- } \alpha & \text { Tumor necrosis factor- } \alpha\end{array}$

\section{References}

1. Eman, E.G.H.; Abdelaziz, M.A.; Taha, N.M.; El-Gama, M.S. The influence of acesulfame-k and aspartame on some physiological parameters in male albino rats. Egypt. J. Hosp. Med. 2019, 75, 1976-1981. [CrossRef]

2. Henry, F.J. Obesity prevention: The key to non-communicable disease control. West Indian Med. J. 2011, 60, 446-451.

3. Hruby, A.; Hu, F.B. The epidemiology of obesity: A big picture. Pharmacoeconomics 2015, 33, 673-689. [CrossRef]

4. Malik, V.S.; Willet, W.C.; Hu, F.B. Nearly a decade on-Trends, risk factors and policy implications in global obesity. Nat. Rev. Endocrinol. 2020, 16, 615-616. [CrossRef] [PubMed]

5. Saris, W.H.M.; Foster, G.D. Simple carbohydrates and obesity: Fact, fiction and future. Int. J. Obes. 2006, 30, S1-S3. [CrossRef]

6. Khaodhiar, L.; McCowen, K.C.; Blackburn, G.L. Obesity and its comorbid conditions. Clin. Cornerstone 1999, 2, 17-31. [CrossRef]

7. Roth, G.A.; Mensah, G.A.; Johnson, C.O.; Addolorato, G.; Ammirati, E.; Baddour, L.M.; Barengo, N.C.; Beaton, A.Z.; Benjamin, E.J.; Benziger, C.P.; et al. Global burden of cardiovascular diseases and risk factors, 1990-2019: Update from the GBD 2019 Study. J. Am. Coll. Cardiol. 2020, 76, 2982-3021. [CrossRef]

8. Tandel, K.R. Sugar substitutes: Health controversy over perceived benefits. J. Pharmacol. Pharmacother. 2011, 2, 236-243. [CrossRef] [PubMed]

9. Walbolt, J.; Koh, Y. Non-nutritive sweeteners and their associations with obesity and type 2 diabetes. J. Obes. Metab. Syndr. 2020, 29, 114-123. [CrossRef] [PubMed]

10. Brown, R.J.; Rother, K.I. Non-Nutritive sweeteners and their role in the gastrointestinal Tract. J. Clin. Endocrinol. Metab. 2012, 97, 2597-2605. [CrossRef]

11. Pang, M.D.; Goossens, G.H.; Blaak, E.E. The impact of artificial sweeteners on body weight control and glucose homeostasis. Front. Nutr. 2021, 7, 598340. [CrossRef] [PubMed]

12. Pearlman, M.; Obert, J.; Casey, L. The association between artificial sweeteners and obesity. Curr. Gastroenterol. Rep. 2017, 19, 64. [CrossRef]

13. Bian, X.; Chi, L.; Gao, B.; Tu, P.; Ru, H.; Lu, K. The artificial sweetener acesulfame potassium affects the gut microbiome and body weight gain in CD-1 mice. PLoS ONE 2017, 12, e0178426. [CrossRef] [PubMed]

14. Zheng, Y.; Sarr, M.G. Effect of the artificial sweetener, acesulfame potassium, a sweet taste receptor agonist, on glucose uptake in small intestinal cell lines. J. Gastrointest. Surg. 2012, 17, 153-158. [CrossRef] [PubMed]

15. Bandyopadhyay, A.; Ghoshal, S.; Mukherjee, A. Genotoxicity testing of low-calorie sweeteners: Aspartame, Acesulfame-K and Saccharin. Drug Chem. Toxicol. 2008, 31, 447-457. [CrossRef]

16. Cong, W.-N.; Wang, R.; Cai, H.; Daimon, C.M.; Scheibye-Knudsen, M.; Bohr, V.A.; Turkin, R.; Wood, W.H.; Becker, K.; Moaddel, R.; et al. Long-Term Artificial Sweetener Acesulfame Potassium Treatment Alters Neurometabolic Functions in C57BL/6J Mice. PLoS ONE 2013, 8, e70257. [CrossRef] 
17. Mukherjee, A.; Chakrabarti, J. In vivo cytogenetic studies on mice exposed to acesulfame-k-A non-nutritive sweetener. Food Chem. Toxicol. 1997, 35, 1177-1179. [CrossRef]

18. Mattes, R.D.; Popkin, B.M. Nonnutritive sweetener consumption in humans: Effects on appetite and food intake and their putative mechanisms. Am. J. Clin. Nutr. 2008, 89, 1-14. [CrossRef]

19. Wang, S.-H.; Yu, T.-Y.; Tsai, F.-C.; Weston, C.J.; Lin, M.-S.; Hung, C.-S.; Kao, H.-L.; Li, Y.-I.; Solé, M.; Unzeta, M.; et al. Inhibition of semicarbazide-sensitive amine oxidase reduces atherosclerosis in apolipoprotein E-deficient mice. Transl. Res. 2018, 197, 12-31. [CrossRef]

20. Wolf, D.; Ley, K. Immunity and inflammation in atherosclerosis. Circ. Res. 2019, 124, 315-327. [CrossRef]

21. Saponaro, C.; Gaggini, M.; Carli, F.; Gastaldelli, A. The Subtle Balance between Lipolysis and Lipogenesis: A Critical Point in Metabolic Homeostasis. Nutrients. 2015, 7, 9453-9474. [CrossRef]

22. Yang, J.; Ma, X.; Li, L.; Wang, L.; Chen, Y.; Liu, J.; Luo, Y.; Zhuang, Z.; Yang, W.; Zang, S.; et al. Berberine ameliorates non alcoholic steatohepatitis in ApoE ${ }^{-/-}$mice. Exp. Ther. Med. 2017, 14, 4134-4140. [CrossRef]

23. Greaves, D.R.; Gordon, S. Immunity, atherosclerosis and cardiovascular disease. Trends Immunol. 2001, 22, 180-181. [CrossRef]

24. Simon, B.R.; Parlee, S.D.; Learman, B.S.; Mori, H.; Scheller, E.; Cawthorn, W.; Ning, X.; Gallagher, K.; Tyrberg, B.; AssadiPorter, F.M.; et al. Artificial sweeteners stimulate adipogenesis and suppress lipolysis independently of sweet taste receptors. J. Biol. Chem. 2013, 288, 32475-32489. [CrossRef]

25. Servant, G.; Kenakin, T.; Zhang, L.; Williams, M.; Servant, N. The function and allosteric control of the human sweet taste receptor. Adv. Pharmacol. 2020, 88, 59-82. [CrossRef]

26. Wauson, E.M.; Lorente-Rodríguez, A.; Cobb, M.H. Minireview: Nutrient sensing by G protein-coupled receptors. Mol. Endocrinol. 2013, 27, 1188-1197. [CrossRef] [PubMed]

27. Barger, P.M.; Brandt, J.M.; Leone, T.C.; Weinheimer, C.J.; Kelly, D.P. Deactivation of peroxisome proliferator-activated receptor- $\alpha$ during cardiac hypertrophic growth. J. Clin. Investig. 2000, 105, 1723-1730. [CrossRef]

28. Simon, B.R.; Learman, B.S.; Parlee, S.D.; Scheller, E.L.; Mori, H.; Cawthorn, W.P.; Ning, X.; Krishnan, V.; Ma, Y.L.; Tyrberg, B.; et al. Sweet taste receptor deficient mice have decreased adiposity and increased bone mass. PLoS ONE 2014, 9, e86454. [CrossRef]

29. Pol, T.; Held, C.; Westerbergh, J.; Lindbäck, J.; Alexander, J.H.; Alings, M.; Erol, C.; Goto, S.; Halvorsen, S.; Huber, K.; et al. Dyslipidemia and risk of cardiovascular events in patients with atrial fibrillation treated with oral anticoagulation therapy: Insights from the ARISTOTLE (apixaban for reduction in stroke and other thromboembolic events in atrial fibrillation) trial. J. Am. Heart Assoc. 2018, 7, e007444. [CrossRef]

30. Veseli, B.E.; Perrotta, P.; De Meyer, G.R.; Roth, L.; Van der Donckt, C.; Martinet, W.; De Meyer, G. Animal models of atherosclerosis. Eur. J. Pharmacol. 2017, 816, 3-13. [CrossRef]

31. Kuipers, F.; Van Ree, J.M.; Hofker, M.H.; Wolters, H.; Veld, G.I.; Havinga, R.; Vonk, R.J.; Princen, H.M.; Havekes, L.M. Altered lipid metabolism in apolipoprotein E-deficient mice does not affect cholesterol balance across the liver. Hepatology 1996, 24, 241-247. [CrossRef]

32. Slätis, K.; Gåfvels, M.; Kannisto, K.; Ovchinnikova, O.; Paulsson-Berne, G.; Parini, P.; Jiang, Z.-Y.; Eggertsen, G. Abolished synthesis of cholic acid reduces atherosclerotic development in apolipoprotein E knockout mice. J. Lipid Res. 2010, 51, 3289-3298. [CrossRef] [PubMed]

33. Jang, W.; Jeoung, N.H.; Cho, K.-H. Modified apolipoprotein (apo) A-I by artificial sweetener causes severe premature cellular senescence and atherosclerosis with impairment of functional and structural properties of apoA-I in lipid-free and lipid-bound state. Mol. Cells 2011, 31, 461-470. [CrossRef] [PubMed]

34. Oppi, S.; Lüscher, T.F.; Stein, S. Mouse models for atherosclerosis research-Which is my line? Front. Cardiovasc. Med. 2019, 6, 46. [CrossRef] [PubMed]

35. Zhao, T.X.; Mallat, Z. Targeting the immune system in atherosclerosis: JASS state-of-the-art review. J. Am. Coll. Cardiol. 2019, 73, 1691-1706. [CrossRef] [PubMed] 